\title{
SPACE CURVES WITH A PRESCRIBED INDEX OF REGULARITY
}

\author{
E. BALLICO \\ Department of Mathematics, University of Trento, 38123 Povo (TN), Italy. \\ E-mail: ballico@science.unitn.it \\ \|\|$\|$
}

\begin{abstract}
We prove the existence of many smooth space curves of degree $d$ and genus $g$ with prescribed index of regularity (we have $g \leq K d^{3 / 2}$ with $K=0.02$ for large $d$ ).
\end{abstract}

\section{Introduction}

Let $X \subset \mathbb{P}^{3}$ be any locally Cohen-Macaulay curve. The index of regularity or regularity index $r(X)$ of $X$ is the minimal integer $t$ such that $h^{1}\left(\mathcal{I}_{X}(x)\right)=0$ for all $x \geq t$, with the convention $r(X)=-\infty$ if $X$ is arithmetically Cohen-Macaulay. The integer $r(X)$ is related to the CastelnuovoMumford regularity of $X$, but it is often easier to compute. The computation of integer $r(X)$ was classically done for special classes of curves, e.g. curves with a singular model with only a small number of singularities or smooth curves on a smooth quadric surface, but the case of space curves seems to be of a different order of difficulty. After [1] we came back to this topic, using the statements and ideas in [1], but not its proof. We state our main result in an axiomatic form (if somebody produce a very good curve $C \subset \mathbb{P}^{3}$, then in the same irreducible component of the Hilbert space of smooth curves there are curves $X \subset \mathbb{P}^{r}$ with certain prescribed $\left.r(X)\right)$. It is [1] which assures the existence of such curves $C$ for many pairs of degree and genera.

Received October 17, 2017

AMS Subject Classification: $14 \mathrm{H} 50$.

Key words and phrases: Space curve, index of regularity, regularity index.

The author was partially supported by MIUR and GNSAGA of INdAM (Italy). 
For any curve $C \subset \mathbb{P}^{3}$ let $N_{C}$ denote the normal sheaf of $C$ in $\mathbb{P}^{3}$ (it is a vector bundle if $C$ is smooth or nodal). In this note we prove the following result.

Theorem 1.1. Fix positive integer $d, m, e, \rho, \delta$ such that $m \geq 13$, e $<m$, $d<\frac{m^{2}+4 m+6}{3}, \rho \geq m+6, \rho+1 \leq \delta \leq \rho+1+\lfloor(\rho-3) / 2\rfloor\lceil m / 6\rceil$ and assume the existence of a smooth and connected curve $C \subset \mathbb{P}^{3}$ such that $\operatorname{deg}(C)=d$, $h^{1}\left(\mathcal{O}_{C}(e)\right)=0, h^{1}\left(N_{C}(-1)\right)=0$ and $h^{1}\left(\mathcal{I}_{C}(t)\right)=0$ for each $t \geq m$. Then there is a smooth and connected curve $X \subset \mathbb{P}^{3}$ with $\operatorname{deg}(X)=d+\delta, p_{a}(X)=$ $p_{a}(C), r(X)=\rho$ and $h^{1}\left(N_{X}(-1)\right)=h^{1}\left(\mathcal{O}_{X}(e)\right)=0$.

The condition " $d<\frac{m^{2}+4 m+6}{3}$ " implies that the pair $(d, m)$ is in the range A in the sense of [4, 5] if we add the condition $h^{0}\left(\mathcal{I}_{C}(m-1)\right)=0$, which we do not impose in Theorem 1.1. We do not assume that $m$ is the minimal integer such that $h^{1}\left(\mathcal{I}_{X}(x)\right)=0$ for all $x \geq m$. The proof works relaxing the assumption $d<\frac{m^{2}+4 m+6}{3}$ at the expense of a stronger assumption on $\delta$. Conversely, stronger upper bounds on $d$ in terms of $m$ (as in [1]) allow any interested reader to weaken the assumption on the upper bound of $\rho$ in terms of $\delta$ and $m$. To get a smooth curve $C \subset \mathbb{P}^{3}$ of degree $d$ and genus $g$ with $h^{1}\left(N_{C}(-1)\right)=0$ (or just with $h^{1}\left(N_{C}\right)=0$ ) we have $d^{3 / 2} / g$ upper bounded for $g \gg 0([3]$, [8, page 11]).

Take $X$ as in Theorem 1.1. Since $h^{1}\left(N_{X}(-1)\right)=0$, we have $h^{1}\left(N_{X}\right)=0$ and hence the Hilbert scheme $\operatorname{Hilb}\left(\mathbb{P}^{3}\right)$ of $\mathbb{P}^{3}$ is smooth at $[X]$ and of dimension $4 \operatorname{deg}(X)$. We will also show how to construct the unique irreducible component of $\operatorname{Hilb}\left(\mathbb{P}^{3}\right)$ containing $X$ if we know the unique irreducible component of $\operatorname{Hilb}\left(\mathbb{P}^{3}\right)$ containing $C$. If $\mu>m$, then $d<\frac{\mu^{2}+4 \mu+6}{3}$ and hence we may use Theorem 1.1 for $C$ and $\mu$. See Lemmas 3.7 and 3.8 for the use of curves $C^{\prime}$ of degree $d^{\prime}>d$ and with genus $p_{a}(C)$ to which we may apply the statement of Theorem 1.1.

Remark 1.2. In Theorem 1.1 set $g:=p_{a}(X)$. By [1, Theorem 1] we may take as $(d, g, m)$ in Theorem 1.1 any triple $(d, g, m)$ with $g \geq 17052$ and $g \leq C m^{3}$ with $C=\sqrt{20} / 600$. We cover in this way the pairs $(d, g)$ for (degree,genus) with $g \leq 0.02 d^{3 / 2}$ and all $d \gg 0([1$, Corollary 1$])$.

We also find irreducible components of the Hilbert scheme of space curves containing two or more curves with prescribed (and different) index of regularity (Remark 3.9). 
We think that the approach used in this paper may be used to attach other problems concerning the Hilbert functions of space curves, but there is a big warning. Since it heavily uses vanishing for the normal bundle of the curve, it may only be used in a range of (degree,genera) $=(d, g)$ with $g / d^{3 / 2}$ upper bounded when $d \gg 0([3],[8, \S 6])$. The general aim is the stratification by degree, genus and Hilbert function of the set of all smooth and connected space curves. In this generality it is an hopeless project for several reasons, but in the Range A something may be done.

To get that a space curve $X \subset \mathbb{P}^{3}$ for which we know that $h^{1}\left(\mathcal{I}_{X}(x)\right)=0$ has $r(X)=x$, we use the following remark (in the set-up of Theorem 1.1 we have $e<m<\rho$ and we take $x=\rho$ ).

Remark 1.3. Let $X \subset \mathbb{P}^{3}$ be a closed subscheme with $\operatorname{dim} X \leq 1$. Fix $x \in \mathbb{N}$ such that $h^{1}\left(\mathcal{I}_{X}(x)\right)=0$ and $h^{1}\left(\mathcal{O}_{X}(x-1)\right)=0$. Since $\operatorname{dim} X \leq 1$, we have $h^{2}\left(\mathcal{O}_{X}(x)\right)=0$ for all $x \in \mathbb{Z}$. Thus $h^{2}\left(\mathcal{I}_{X}(x-1)\right)=0$. The Castelnuovo-Mumford's lemma gives $h^{1}\left(\mathcal{I}_{X}(t)\right)=0$ for all $t>x$. Thus if $h^{1}\left(\mathcal{I}_{X}(x-1)\right) \neq 0$ the integer $x$ is the index of regularity of $X$. To get $h^{1}\left(\mathcal{I}_{X}(x-1)\right)$ we will construct $X$ such that $h^{2}\left(\mathcal{I}_{X}(x-2)\right)=0$ and there is a line $D \subset \mathbb{P}^{3}$ with $\operatorname{deg}(D \cap X)=x+1$. Since every $G \in\left|\mathcal{I}_{X}(x)\right|$ contains $D$ and $D \nsubseteq X$, the homogeneous ideal of $X$ is not generated by forms of degree at most $x$. Since $h^{2}\left(\mathcal{I}_{X}(x-2)\right)=0$, the Castelnuovo-Mumford's lemma gives $h^{1}\left(\mathcal{I}_{X}(x-1)\right) \neq 0$.

By Remark 1.3 the general strategy is to construct a pair $(X, D)$, where $X$ is a smooth curve of genus $g$ and degree $d$ with $h^{1}\left(\mathcal{I}_{X}(\rho)\right)=0$ and $D$ is a line with $\operatorname{deg}(X \cap D)=\rho+1$. In the set-up of Theorem 1.1 we take as $X$ a very special smoothing (we need a line $D$ with $\operatorname{deg}(X \cap D)=\rho+1$ ) of a union of $C$ and $d-\delta$ lines.

\section{The Components of the Hilbert Schemes in which We Land}

Lemma 2.1. Let $C \subset \mathbb{P}^{3}$ be a smooth curve with $h^{1}\left(N_{C}(-1)\right)=0$. Let $D \subset \mathbb{P}^{3}$ be a smooth rational curve intersecting $C$ at a unique point, $q$, and quasi-transversally. Then $C \cup D$ is smoothable and $h^{1}\left(N_{C \cup D}(-1)\right)=0$. If e is a positive integer with $h^{1}\left(\mathcal{O}_{C}(e)\right)=0$, then $h^{1}\left(\mathcal{O}_{C \cup D}(e)\right)=0$. 
Proof. The curve $C \cup D$ is smoothable by [7, Theorem 4.1] or [9, Proposition $1.6]$.

Let $N$ be the vector bundle on $C$ obtained from $N_{C}$ making a positive elementary transformation at $q$ in the direction of the tangent line of $D$ at $q$. Let $M$ be the vector bundle on $D$ obtained from $N_{D}$ making a positive elementary transformation at $q$ in the direction of the tangent line of $C$ at $q$. We have $N=N_{C \cup D \mid C}$ and $M=N_{C \cup D \mid D}$ ([7, Corollary 3.2]). Thus we have the Mayer-Vietoris exact sequence

$$
0 \rightarrow N_{C \cup D}(-1) \rightarrow N(-1) \oplus M(-1) \rightarrow M(-1)_{\mid\{q\}} \rightarrow 0
$$

Since $h^{1}\left(N_{C}(-1)\right)=0$ and $N_{C}(-1)$ is a subsheaf of $N(-1)$ such that $N(-1) / N_{C}(-1)$ has zero-dimensional support, we have $h^{1}(M(-1))=0$. If $M(-1)$ is spanned, then the restriction map $H^{0}(M(-1)) \rightarrow H^{0}\left(M(-1)_{\mid\{q\}}\right)$ is surjective and therefore the map $H^{0}(N(-1) \oplus M(-1)) \rightarrow H^{0}\left(M(-1)_{\mid\{q\}}\right)$ induced by (11) is surjective. By (1) to prove that $h^{1}\left(N_{C \cup D}(-1)\right)=0$ it is sufficient to prove that $h^{1}(M(-1))=0$ and that $M(-1)$ is spanned. Since $D$ is smooth, $N_{D}$ is a quotient of $T \mathbb{P}_{\mid D}^{3}$, sequence of $T \mathbb{P}^{3}$ gives a surjection $\mathcal{O}_{\mathbb{P}^{3}}^{\oplus 4} \rightarrow T \mathbb{P}^{3}$. Restricting to $D$ we get that $N_{D}(-1)$ is spanned. Thus by the classification of vector bundles on $\mathbb{P}^{1}$ the vector bundle $N_{D}(-1)$ is a direct sum of 2 line bundles of degree $\geq 0$. Hence $M(-1)$ is a direct sum of line bundles of degree $\geq 0$. Thus $h^{1}(M(-1))=0$ and $M(-1)$ is spanned.

Since $\mathcal{O}_{D}(e)$ is spanned, the Mayer-Vietoris exact sequence

$$
0 \rightarrow \mathcal{O}_{C \cup D}(e) \rightarrow \mathcal{O}_{C}(e) \oplus \mathcal{O}_{D}(e) \rightarrow \mathcal{O}_{q}(e) \rightarrow 0
$$

gives $h^{1}\left(N_{C \cup D}(e)\right)=0$.

Notation 2.2. Let $\Gamma$ be an irreducible component of Hilb $\left(\mathbb{P}^{3}\right)$ whose general element is a smooth and connected curve $C$ with $h^{1}\left(N_{C}(-1)\right)=0$. For each integer $t>0$ let $\Gamma_{t}$ be the only irreducible component of $\operatorname{Hilb}\left(\mathbb{P}^{3}\right)$ containing $C \cup D$, where $D$ is a smooth rational curve of degree $t$ intersecting transversally $C$ at a unique point (Lemma 2.1). We have $h^{1}\left(N_{X}(-1)\right)=0$ for a general $X \in \Gamma_{t}$ by Lemma 2.1.

Lemma 2.3. If $t \geq 2$, then $\Gamma_{t}=\left(\Gamma_{1}\right)_{t-1}$. 
Proof. Take $C \in \Gamma$ with $h^{1}\left(N_{C}(-1)\right)=0$ and a smooth degree $t$ rational curve $D$ with $\sharp(D \cap C)=1$, say $C \cap D=\{q\}$, and intersecting quasitransversally $C$ at $q$. We may degenerate $D$ in a family of curves containing $q$ to a curve $R \cup A$ with $R$ a line, $q \in R, R$ is not the tangent line of $C$ at $q, R \cap C=\{q\}, A$ is a degree $t-1$ smooth rational curve with $A \cap$ $C=\emptyset, A$ intersects $R$ at a single point, $a$, and quasi-transversally. Since $h^{1}\left(N_{C \cup R \cup A}\right)=0$ (easier that the case done in the proof of Lemma 2.1) , there is a unique irreducible component $\Psi$ of $\operatorname{Hilb}\left(\mathbb{P}^{3}\right)$ containing $C \cup R \cup A$. By the definition of $\Gamma_{t}$ and the fact that $C \cup R \cup A$ is a degeneration of $C \cup D$, this component is just $\Gamma_{t}$. We have $C \cup R \in \Gamma_{1}$. Since $h^{1}\left(N_{C \cup R}(-1)\right)=0$ (Lemma 2.1), we have $h^{1}\left(N_{B}(-1)\right)=0$ for a general $B \in \Gamma_{1}$. Let $\pi: \mathcal{X} \rightarrow \Delta$ be a flat family with special fiber $\pi^{-1}(o)$ and $B=\pi^{-1}(b)$ as a general fiber. Since $a$ is a smooth point of $C \cup R$, up to a finite covering of $\Delta$ we may find a section $u$ of $\pi$ with $u(o)=a$. Up to a quasi-finite covering of $\Delta$ with image containing both $o$ and $b$ we may find a family of smooth degree $t-1$ rational curves $\eta: \mathcal{D} \rightarrow \Delta$ and a section $v$ of $\eta$ with $\eta^{-1}(o)=A, \eta^{-1}(b) \cap B=v(b)$, $\eta^{-1}(b)$ and $B$ are quasi-transversal and $v(o)=a$. The curve $B \cup \eta^{-1}(b)$ is in $\left(\Gamma_{1}\right)_{t-1}$.

Remark 2.4. Take a smooth and connected curve $C \subset \mathbb{P}^{3}$, an integer $t \geq 2$, and lines $L_{i} \subset \mathbb{P}^{3}, 1 \leq i \leq t$, such that $L_{1}$ intersects $C$ quasitransversally and at a unique point, while for all $i=2, \ldots, t$ the line $L_{i}$ meets $C \cup L_{1} \cup \cdots \cup L_{i-1}$ quasi-transversally and at a unique point. As in the proof of Lemma 2.3 we see that $C \cup L_{1} \cup \cdots \cup L_{t} \in \Gamma_{t}$.

\section{The index of regularity}

For any closed subscheme $Z \subset \mathbb{P}^{3}$ and any plane $H \subset \mathbb{P}^{3}$ the residual scheme of $Z$ with respect to $H$ is the closed subscheme of $\mathbb{P}^{3}$ with $\mathcal{I}_{Z}: \mathcal{I}_{H}$ as its ideal sheaf. We have an exact sequence

$$
0 \rightarrow \mathcal{I}_{\operatorname{Res}_{H}(Z)}(t-1) \rightarrow \mathcal{I}_{Z}(t) \rightarrow \mathcal{I}_{Z \cap H, H}(t) \rightarrow 0
$$

For any $o \in \mathbb{P}^{3}$ let $2 o$ denote the first infinitesimal neighborhood of $o$ in $\mathbb{P}^{3}$, i.e. the closed subscheme of $\mathbb{P}^{3}$ with $\left(\mathcal{I}_{o}\right)^{2}$ as its ideal sheaf (it is a zero-dimensional scheme with degree 3 and $\left.2 o_{\text {red }}=\{o\}\right)$. If $H \subset \mathbb{P}^{3}$ is a plane with $o \in H$, then $\operatorname{Res}_{H}(2 o)=\{o\}$ and $2 o \cap H$ is the first infinitesimal 
neighborhood of $o$ in $H$. Hence for any scheme $T \subset H$, then $\operatorname{Res}_{H}(T \cup 2 o)=$ $\{o\}$. If $T$ is a curve, which is singular at $\{o\}$, then $(T \cup 2 o) \cap H=T$.

Lemma 3.1. Let $C \subset \mathbb{P}^{3}$ be a smooth and connected curve with $h^{1}\left(N_{C}(-1)\right)$ $=0$. Let $H \subset \mathbb{P}^{3}$ be a plane intersecting transversally $C$. Fix $S \subsetneq C \cap H$, $o \in H \cap C \backslash S$ and a general $q \in H$. Let $D \subset H$ be the line spanned by $o$ and $q$. Then $C \cup D$ is smoothable, $h^{1}\left(N_{C \cup D}(-1)\right)=0$ and there are $a$ flat family $\left\{X_{t}\right\}_{t \in \Delta}$ of space curves and $a \in \Delta$ with $\Delta$ an irreducible curve, $X_{a}=C \cup D, X_{t}$ smooth if $t \neq a$ and $S \cup\{q\} \subset X_{t}$ for all $t \in \Delta$.

Proof. Concerning the point $q$ we only need $q \neq o$ (to define $D$ ) and that the line $D$ meets $C$ only at $o$. Set $w:=\sharp(S)$. By Lemma $2.1 Y:=C \cup D$ is smoothable and $h^{1}\left(N_{Y}(-1)\right)=0$. We need to prove that $Y$ is smoothable in a family of space curves all of them containing $S \cup\{q\}$. Since $S \cup\{o\} \subset H$, we have $h^{1}\left(N_{C}(S-\{o\}) \leq h^{1}\left(N_{C}(-1)\right)=0\right.$. Since $N_{D} \cong \mathcal{O}_{D}(1)^{\oplus 2}$, we have $h^{1}\left(N_{D}(-o-q)\right)=0$ and $N_{D}(-q) \cong \mathcal{O}_{D}^{\oplus 2}$. Since $Y \cup D$ is nodal the MayerVietoris exact sequence of $Y=C \cup D$ gives $h^{1}\left(N_{Y}(-(S \cup\{q\}))=0\right.$. Let $\pi: W \rightarrow \mathbb{P}^{3}$ be the blowing up of $\mathbb{P}^{3}$ along $S \cup\{q\}$. For any curve $E \subset W$ let $N_{E, W}$ denote the normal sheaf of $E$ in $W$. Let $Y^{\prime}, C^{\prime}$ and $D^{\prime}$ be the strict transforms of $Y, C$ and $D$ in $W$. Since $Y$ is smooth at all points of $S \cup\{q\}$, $\pi$ induces an isomorphism between $Y^{\prime}, C^{\prime}, D^{\prime}$ and $Y, C, D$, respectively and this isomorphism maps $N_{Y}(-(S \cup\{q\})), N_{C}(-S), N_{D}(-q)$ isomorphically onto $N_{Y^{\prime}, W}, N_{C^{\prime}, W}$ and $N_{D^{\prime}, W}$. Hence $H^{0}\left(N_{Y}(-S-\{q\})\right)$ is the tangent space at $C \cup D$ of the deformation functor of the curves containing $S \cup\{q\}$, while $H^{1}\left(N_{Y}(-S-\{q\})\right)$ is an obstruction space for the same functor (see [8] for several uses of this set-up). At this point we have basically won, but we show how to adapt the proof in [7, Theorem 4.1] to conclude the proof.

Claim 1: $Y^{\prime}$ is smoothable inside $W$.

Proof of Claim 1. $Y^{\prime}$ has a unique singular point, the only point $o^{\prime} \in W$ with $\pi\left(o^{\prime}\right)=o$, and the curves $C^{\prime}$ and $D^{\prime}$ meets transversally at $o^{\prime}$. Since $D \cap(S \cup\{q\})=q$ and $N_{D} \cong \mathcal{O}_{D}^{\oplus 2}(1), N_{D^{\prime}, W} \cong \mathcal{O}_{D^{\prime}}^{\oplus 2}$. Hence $h^{1}\left(D^{\prime}, \mathcal{W}\right)=$ 0 for any sheaf $\mathcal{W}$ obtained from $N_{D^{\prime}, W}$ making one negative elementary transformation at $o^{\prime}$. Since $h^{1}\left(N_{C}(-1)\right)=0$ and $S \subseteq C \cap H$, we have $h^{1}\left(N_{C}(-S)\right)=0$, i.e. $h^{1}\left(N_{C^{\prime}, W}\right)=0$. Apply [7, Theorem 4.1].

Since $h^{1}\left(N_{Y^{\prime}}\right)=0$, the Hilbert scheme of $W$ is smooth at $Y^{\prime}$. Call $X^{\prime}$ any smooth curve belonging to the irreducible component of the Hilbert scheme 
of $W$ containing $Y^{\prime}$ (it exists by Claim 1). $W$ has $w+1$ exceptional divisors, i.e. smooth rational curves with self-intersection -1 mapped by $\pi$ to different points of $S \cup\{q\}$. we have $\operatorname{Pic}(W) \cong \mathbb{Z}^{w+2}$ with as free generators $\pi^{*}\left(\mathcal{O}_{\mathbb{P}^{3}}(1)\right)$ and these $w+1$ exceptional divisors. Let $J$ be one these exceptional divisors, say $J=\pi^{-1}(a)$ with $a \in S \cup\{q\}$. Since $a$ is a smooth point of $Y$, we have $\operatorname{deg}\left(Y^{\prime} \cap J\right)=1$ and hence $Y^{\prime} \cdot J=1$ in the intersection ring of $J$. Hence $Y^{\prime} \cap J=1$. Thus $a \in \pi\left(X^{\prime}\right)$. Thus $\pi\left(X^{\prime}\right) \supset S \cup\{q\}$. Since $Y^{\prime} \cdot J=1, Y^{\prime}$ intersects transversally $J$ at a unique point. Since $Y^{\prime}$ is smooth, we get that $\pi\left(X^{\prime}\right)$ is smooth at $a$ for all $a \in S \cup\{q\}$, concluding the proof of the lemma.

Remark 3.2. For any non-degenerate, reduced and connected $C \subset \mathbb{P}^{3}$ and any plane $H \subset \mathbb{P}^{3}$ the scheme $C \cap H$ spans $H$ (use the the exact sequence

$$
0 \rightarrow \mathcal{I}_{C} \rightarrow \mathcal{I}_{C}(1) \rightarrow \mathcal{I}_{C \cap H, H}(1) \rightarrow 0
$$

and that $h^{1}\left(\mathcal{I}_{C}\right)=0$, because $\left.h^{0}\left(\mathcal{O}_{C}\right)=1\right)$. Hence if $H$ intersects transversally $C$ and $S \subseteq C \cap H$ is formed by collinear points, then we have $S \neq C \cap H$ and hence we may find $o \in C \cap H \backslash H$ to which we apply the statement of Lemma 2.1.

Lemma 3.3. Let $C \subset \mathbb{P}^{3}$ be a smooth and connected curve with $h^{1}\left(N_{C}(-1)\right)$ $=0$. Let $H \subset \mathbb{P}^{3}$ be a plane intersecting transversally $C$. Fix an integer $t>0, t$ distinct points $p_{1}, \ldots, p_{t} \in H \cap C$ and $t$ distinct points $o_{1}, \ldots, o_{t} \in$ $H \backslash H \cap C$. Let $L_{i}$ be the line spanned by $\left\{o_{i}, p_{i}\right\}$. Assume that all lines $L_{i}$ are distinct, no point of $H$ is contained in 3 of them and that $\left\{p_{1}, \ldots, p_{t}\right\}=$ $C \cap T$, where $T:=\cup_{i=1}^{t} L_{i}$. Set $E:=\operatorname{Sing}(T)$ and $\chi:=\cup_{o \in E} 2 o$. Fix a set $S \subseteq C \cap H \backslash T \cap C$. Then $C \cup T \cup \chi$ is smoothable in a family of curves all containing $\left\{o_{1}, \ldots, o_{t}\right\} \cup S$.

Proof. The case $t=1$ is true by Lemma 3.1. Now assume $t>1$ and that the lemma is true for the integer $t-1$. Set $T^{\prime}:=L_{1} \cup \cdots \cup L_{t-1}$ and $\chi^{\prime}:=\operatorname{Sing}\left(T^{\prime}\right)$. By the inductive assumption $C \cup T^{\prime} \cup \chi^{\prime}$ is smoothable in a family of curves containing $A \cup\left\{o_{1}, \ldots, o_{t}\right\}$ and whose general member $Y$ satisfies $h^{1}\left(N_{Y}(-1)\right)=0$. There are an integral affine curve, a family of lines $\left\{R_{b}\right\}_{b \in \Delta}$ of $\mathbb{P}^{3}$ and $a \in \Delta$ such that $R_{a}=L_{t}, R_{b}$ meets $C$ at a unique point, $u_{b}$, and quasi-transversally and $u_{b} \notin H$ for all $b \neq a$. Obviously $C \cup T \cup \chi$ is a degeneration of the family $\left\{C \cup T^{\prime} \cup R_{b}\right\}_{b \in \Delta \backslash\{a\}}$. Hence $C \cup T \cup \chi$ is smoothable. But we also need to check that it is smoothable preserving 
$A \cup\left\{o_{1}, \ldots, o_{t}\right\}$ and that a general smoothing $Y$ has $h^{1}\left(N_{Y}(-1)\right)=0$. The scheme $C \cup T \cup \chi$ is a flat limit of a family of nodal curves $\left\{W_{a}\right\}_{a \in \Delta}$ with $W_{b}=C \cup T \cup \chi$ for some $b \in \Delta$ and $W_{a}=C \cup D_{1}(a) \cup \cdots \cup D_{t}(a)$ a connected nodal curve, each $D_{i}(a)$ a line intersecting quasi-transversally $C$ and at a unique point $p_{i}(a) \in C \backslash C \cap H$, each $D_{i}(a)$ containing $o_{i}$ and $D_{i}(a) \cap D_{j}(a)=$ $\emptyset$ for all $i \neq j$ and all $a \in \Delta \backslash\{a\}$ (use that through two different points of $\mathbb{P}^{3}$ there is a unique line and that this line depends regularly on the points). Fix a general $a \in \Delta$ and set $F:=D_{1}(a) \cup \cdots \cup D_{t}(a), q_{i}:=p_{i}(a)$ and $W:=W_{a}$. Note that $W=C \cup F$ and $C \cap F\left\{q_{1}, \ldots, q_{t}\right\}=\operatorname{Sing}(W)$. Since $W$ is nodal, $N_{W}(-1)$ is locally free. Thus we have the Mayer-Vietoris exact sequence

$$
0 \rightarrow N_{W}(-1) \rightarrow N_{W}(-1)_{\mid C} \oplus N_{W}(-1)_{\mid F} \rightarrow N_{W}(-1)_{\left\{q_{1}, \ldots, q_{t}\right\}} \rightarrow 0
$$

The restriction of $N_{W}(-1)$ to each connected component of $F$ is the direct sum of a line bundle of degree 1 and a line bundle of degree 0 . Thus the restriction map $H^{0}\left(N_{W}(-1)_{\mid F}\right) \rightarrow H^{0}\left(N_{W}(-1)_{\left\{q_{1}, \ldots, q_{t}\right\}}\right\}$ is surjective. Therefore (11) gives $h^{1}\left(N_{W}(-1)\right)=0$. By the semicontinuity theorem for cohomology we get the lemma.

Lemma 3.4. Let $T=L_{1} \cup \cdots \cup L_{b} \subset \mathbb{P}^{2}, b \geq 2$, be a nodal union of $b$ distinct lines. Let $E \subset \mathbb{P}^{2}$ be the union of all points $L_{i} \cap L_{j}$ with $i \geq j+2$. Then $h^{1}\left(\mathbb{P}^{2}, \mathcal{I}_{E}(b-3)\right)=0$.

Proof. If $b=2$, then $E=\emptyset$ and hence the lemma is true in this case. Now assume $b>2$ and that lemma is true for a smaller number of lines. We have $\sharp\left(L_{b} \cap E\right)=b-2$ and hence $h^{1}\left(L_{b}, \mathcal{I}_{E \cap L_{b}, L_{b}}(b-3)\right)=0$. Use the residual exact sequence of sheaves on $\mathbb{P}^{2}$ :

$$
0 \rightarrow \mathcal{I}_{E \backslash E \cap L_{b}}(b-4) \rightarrow \mathcal{I}_{E}(b-3) \rightarrow \mathcal{I}_{E \cap L_{b}, L_{b}}(b-3) \rightarrow 0
$$

and the inductive assumption.

Lemma 3.5. Let $C \subset \mathbb{P}^{3}$ be a smooth curve with $h^{1}\left(N_{C}(-1)\right)=0$. Let $L \subset \mathbb{P}^{3}$ be a line with $\sharp(L \cap C)=1$ and $L$ intersecting quasi-transversally C. Set $\{q\}:=L \cap C$. Fix a line $D$ intersecting quasi-transversally $C$, with $q \notin D$ and with $D \cap L \neq \emptyset$. Then there is a smoothing of $Y:=C \cup L$ in a family of curves all containing the set $S:=(C \cup L) \cap D$. 
Proof. Set $\{o\}:=L \cap D$ and $S^{\prime}:=S \backslash\{o\}$. Let $\pi: W \rightarrow \mathbb{P}^{3}$ be the blowing up of $S$. Let $C^{\prime}, L^{\prime}$ and $Y^{\prime}$ be the strict transform in $W$ of $C$, $L$ and $Y$. Since $C, L$ and $Y$ are smooth at the points of $S, \pi$ induces an isomorphism between $C^{\prime}$ (resp. $L^{\prime}$, resp. $\left.Y^{\prime}\right)$ and $C$ (resp. $L$, resp. $Y$ ) and this isomorphism induces an isomorphism between $N_{C^{\prime}}$ (resp. $N_{L^{\prime}}$, resp. $N_{Y^{\prime}}$ ) and $N_{C}\left(-S^{\prime}\right)$ (resp. $N_{L}(-o)$, resp. $N_{Y}(-S)$ ). To prove the lemma it is sufficient to smooth $Y^{\prime}$ in $W$, because in the smoothing family each element would intersect each exceptional divisor of $\pi$. Since $S^{\prime} \subset D, D$ is contained in a plane and $h^{1}\left(N_{C}(-1)\right)=0$, we have $h^{1}\left(N_{C}\left(-S^{\prime}\right)\right)=0$, i.e. $h^{1}\left(N_{C^{\prime}}\right)=0$. We have $N_{L}(-o) \cong \mathcal{O}_{L}^{\oplus 2}$, i.e. $N_{L^{\prime}} \cong \mathcal{O}_{L^{\prime}}^{\oplus 2}$. Hence the vector bundle $M$ on $L^{\prime}$ obtained from $N_{L^{\prime}}$ making a negative elementary transformation is a direct sum of a line bundle of degree -1 and a line bundle of degree 0 . Thus $h^{1}(M)=0$. The smoothing of $Y^{\prime}$ is obtained as in the proof of [7, Theorem 4.1].

Lemma 3.6. Fix an integer $b>0$. Let $C \subset \mathbb{P}^{3}$ be a smooth curve with $h^{1}\left(N_{C}(-1)\right)=0$. Let $D, L_{1} \subset \mathbb{P}^{3}$ be lines with $\sharp\left(L_{1} \cap C\right)=\sharp(D \cap C)=$ $\sharp\left(D \cap L_{1}\right)=1, L_{1}$ and $D$ intersecting quasi-transversally $C$ and $D \cap C \notin L_{1}$. If $b \geq 2$ define recursively the lines $L_{i}, 2 \leq i \leq b$, in the following way. Let $L_{i}, 2 \leq i \leq b$, be a general line intersecting both $L_{i-1}$ and $D$. The curve $Y:=C \cup L_{1} \cup \cdots \cup L_{b}$ is nodal and connected and it may be smoothed in a family of curves all containing the set $D \cap\left(C \cup L_{1} \cup \cdots \cup L_{b}\right)$.

Proof. The case $b=1$ is true by Lemma 3.5. Now assume $b>1$ and that the lemma is true for a smaller number of lines. By Lemma 3.5 there is a smoothing $\left\{X_{a}\right\}_{a \in \Delta}$ of $C \cup L_{1}$ (say $X_{o}=C \cup L_{1}$ with $o \in \Delta$ ) in a family of curves containing $D \cap\left(C \cup L_{1}\right)$. By assumption the point $b:=L_{1} \cap L_{2}$ is a smooth point of $C \cup L_{1}$ and hence, taking if necessary a finite covering of $\Delta$, there is a section $u$ of $\left\{X_{a}\right\}_{a \in \Delta}$ with $u(o)=b$. Taking a smaller $\Delta$ if necessary we may assume that for every $a \in \Delta$ we have $u(a) \notin D \cup C$ and the line $L_{2}(a)$ spanned by $u(a)$ and $D \cap L_{2}$ does not intersects $C$. We take a general $a \in \Delta$ and apply Lemma 3.5 to $X_{a} \cup L_{2}(a)$ to get the case $b=2$; call $\left\{T_{c}\right\}_{c \in \Theta}$ this smoothing and $o^{\prime} \in \Theta$ with $T_{o^{\prime}}$ smooth. Now assume $b>2$. Since any 2 points of $\mathbb{P}^{3}$ span a line, we may find an equisingular deformation $\left\{L_{3}(a) \cup \cdots \cup L_{b}(a)\right\}_{a \in \Delta}$ of $L_{3} \cup \cdots \cup L_{b}$ with $L_{3}(o) \cup \cdots \cup L_{b}(o)=L_{3} \cup \cdots \cup L_{b}$ and $L_{3}(a)$ meeting $L_{2}(a)$ at a unique point and then (for a fixed general $a \in \Delta$ ) an equisingular deformation $\left\{R_{3}(c) \cup \cdots \cup R_{b}(c)\right\}_{c \in \Theta}, o^{\prime} \in \Theta$, with 
$R_{3}\left(o^{\prime}\right) \cup \cdots \cup R_{b}\left(o^{\prime}\right)=L_{3}(a) \cup \cdots \cup L_{3}(a)$ with $R_{3}(c)$ intersecting quasitransversally $T_{c}$ and at a unique point for all $c \in \Theta$. Thus we conclude by the inductive assumption.

Lemma 3.7. Fix positive integers $m, d$ and a smooth and connected curve $C \subset \mathbb{P}^{3}$ such that $h^{1}\left(N_{C}(-1)\right)=0, h^{1}\left(\mathcal{O}_{C}(e)\right)=0, h^{1}\left(\mathcal{I}_{C}(x)\right)=0$ for all $x \geq m$ and $d:=\operatorname{deg}(D) \leq\left(m^{2}+m+2\right) / 2$. Let $F$ be either a line or a smooth conic. Assume $\sharp(C \cap F)=1$ and that $F$ intersects quasi-transversally $C$. Set $Y:=C \cup F$. Then $Y$ is smoothable, $p_{a}(Y)=p_{a}(C), h^{1}\left(N_{Y}(-1)\right)=0$ and $h^{1}\left(\mathcal{I}_{Y}(x)\right)=0$ for all $x \geq m+1$.

Proof. By Lemma 2.1 it is sufficient to prove the last assertion. Let $H$ be a plane containing $F$. Since $h^{1}\left(N_{C}(-1)\right)=0$, we may deform $C$ in a family of curves containing the point $C \cap F$ so that (after this deformation), $C \cap(H \backslash F)$ is a general union of $d-1$ points of $H$. From the residual exact sequence of $\mathcal{I}_{Y}$ with respect to $H$ we get the exact sequence

$$
0 \rightarrow \mathcal{I}_{C}(t-1) \rightarrow \mathcal{I}_{Y}(t) \rightarrow \mathcal{I}_{F \cup(C \cap(H \backslash F), H}(t) \rightarrow 0
$$

Since $d-1 \leq(m+1) m / 2$ and $C \cap(H \backslash F)$ is general in $H$, we have $h^{1}\left(H, \mathcal{I}_{D \cup(C \cap(H \backslash D), H}(t)\right)=0$ for all $t>m$. From (2) we get $h^{1}\left(\mathcal{I}_{Y}(x)\right)=0$ for all $x \geq m+1$.

Lemma 3.8. Fix positive integers $m, e, b, d$ with $b \geq 1, d-1 \leq\left(\begin{array}{c}m+4-b \\ 2\end{array}\right)$ and $b<m$. Let $C$ be a smooth and connected curve $C \subset \mathbb{P}^{3}$ such that $\operatorname{deg}(C)=d, h^{1}\left(N_{C}(-1)\right)=0, h^{1}\left(\mathcal{O}_{C}(e)\right)=0, h^{1}\left(\mathcal{I}_{C}(x)\right)=0$ for all $x \geq m$. Let $Y=C \cup L_{1} \cup \cdots \cup L_{b}$ be a general union of $C$, a general line $L_{1}$ intersecting $C$ and, for $i=2, \ldots, b$, a general line $L_{i}$ intersecting $L_{i-1}$. Then $Y$ is smoothable $h^{1}\left(N_{Y}(-1)\right)=0$ and $h^{1}\left(\mathcal{I}_{Y}(x)\right)=0$ for all $x \geq m+2$.

Proof. As in Lemma 3.6 we see that $Y$ is smoothable and that $h^{1}\left(N_{Y}(-1)\right)$ $=0$. We use a degeneration of $Y$ and the semicontinuity theorem to prove that $h^{1}\left(\mathcal{I}_{Y}(x)\right)=0$ for all $x \geq m+2$. Set $R_{1}:=L_{1}$ and take a plane $H \supset R_{1}$. Since $h^{1}\left(N_{C}(-1)\right)=0$, we may deform $Y$ keeping fixed the point $\{q\}:=R_{1} \cap C$ so that the other $d-1$ points of $C \cap H$ are general in $H$. Fix general lines $R_{2}, \ldots, R_{b}$ of $H$ and let $E$ be the union of the points $R_{i} \cap R_{j}$ with $i \geq j+2$. We have $\sharp(E)=(b-1)(b-2) / 2$. Set $\chi:=\cup_{o \in E} 2 o$, $T:=R_{1} \cup \cdots \cup R_{b}$ and $M:=C \cup T \cup \chi$. As in the proof of Lemma 3.3 we see by induction on $b$ (using [2] or [6, Example 2.1.1] at each point of $E$ ) that 
$M$ is a flat limit of connected nodal curves with arithmetic genus $p_{a}(C)$ like $C \cup D_{1} \cup \cdots \cup D_{b}$ with $D_{1}, \ldots, D_{b}$ lines, $C \cap D_{1} \neq \emptyset$ and $D_{i} \cap D_{j} \neq \emptyset$ if and only if $|i-j| \leq 1$. We have the residual exact sequences of $\mathcal{I}_{C \cup E}$ and of $\mathcal{I}_{M}$ with respect to $H$ :

$$
\begin{aligned}
& 0 \rightarrow \mathcal{I}_{C}(x-2) \rightarrow \mathcal{I}_{C \cup E}(x-1) \rightarrow \mathcal{I}_{E \cup(C \cap E), H}(x-1) \rightarrow 0, \\
& 0 \rightarrow \mathcal{I}_{C \cup E}(x-1) \rightarrow \mathcal{I}_{M}(x) \rightarrow \mathcal{I}_{T \cup(C \cap(H \backslash\{q\}, H}(x) \rightarrow 0 .
\end{aligned}
$$

For each $x \geq m+2$ we have $h^{1}\left(H, \mathcal{I}_{T \cup(C \cap(H \backslash\{q\}, H}(x)\right)=h^{1}\left(H, \mathcal{I}_{(C \cap H) \backslash\{q\}}(x+\right.$ $2-b))=0$ (by the assumption $\left.d-1 \leq\left(\begin{array}{c}m+4-b \\ 2\end{array}\right)\right)$ and $h^{1}\left(H, \mathcal{I}_{E \cup(C \cap E), H}(x-\right.$ 1) $)=0$ (because $h^{1}\left(H, \mathcal{I}_{E}(b-3)\right)=0, b \leq m, C \cap H$ is general in $H$ and $d+(b-1)(b-2) / 2 \leq\left(\begin{array}{c}m+3 \\ 2\end{array}\right)$.

For any positive integer $x \geq 1$ a chain of $x$ lines is a reduced, connected and nodal curve $F \subset \mathbb{P}^{3}$ such that $F=L_{1} \cup \cdots \cup L_{x}$ with each $L_{i}$ a line, $L_{i} \neq L_{j}$ for all $i \neq j$ and $L_{i} \cap L_{j} \neq \emptyset$ if and only if $|i-j| \leq 1$. Since each irreducible component of $F$ is a line, if $|i-j|=1$, then $L_{i}$ intersect quasi-transversally $L_{j}$ and at a unique point. We have $p_{a}(F)=0$ and $F$ is smoothable to a smooth rational curve of degree $x$ ([7]), but we need more if $x \geq 2$. Now assume $x \geq 2$ and fix $o \in L_{1} \backslash L_{1} \cap L_{2}$. Since $F$ is nodal, $N_{F}$ is a rank 2 vector bundle. As in []] we see that $N_{F \mid L_{i}}$ is the direct sum of two line bundles of degree $\geq 1$. As in the proof of Claim 1 in the proof of Lemma 3.1 we see that $F$ may be smoothed in a family of space curves containing $o$.

Proof of Theorem 1.1. Call $\Gamma$ the irreducible component of the Hilbert scheme of $\mathbb{P}^{3}$ containing $C$. We will get $X \in \Gamma_{\delta}$.

(a) In this step we assume $\rho-m$ even and $\delta:=\rho+1$. Set $c:=(\rho-m) / 2$.

Fix a line $D \subset \mathbb{P}^{3}$ such that $C \cap D=\emptyset$ and distinct planes $H_{i}, i \geq 1$, containing $D$. Note that $D=H_{i} \cap H_{j}$ for all $i \neq j$. Let $a_{1}$ be the maximal integer $x$ such that $d-1 \leq \underset{2}{m+3-x})$. We have $a_{1} \geq 2$, because $d \leq m^{2} / 2$. Set $b_{1}:=\min \left\{a_{1}, \delta\right\}$. Take an integer $i \geq 2$ and assume defined the integers $a_{j}$ and $b_{j}$ for all positive integers $j<i$. Let $a_{i}$ be the maximal integer $x$ such that $\left(\begin{array}{c}m+2 i+1-x \\ 2\end{array}\right) \geq d-1+b_{1}+\cdots+b_{i-1}$. Set $b_{i}:=\min \left\{a_{i}, m+2 i+\right.$ $\left.1-b_{1}-\cdots-b_{i-1}\right\}$. We obviously have $a_{i} \leq m+2 i-1$ for all $i$. Note that $b_{1}+\cdots+b_{x}=\rho+1=\delta$ for $x \gg 0$. If there is an integral curve $T \subset \mathbb{P}^{3}$ with $h^{1}\left(\mathcal{O}_{T}(\rho-1)\right)=0$ and $\operatorname{deg}(T \cap D) \geq \rho+1$, then $h^{1}\left(\mathcal{I}_{T}(\rho-1)\right)>0$ 
by the Castelnuovo-Mumford lemma, because the homogeneous ideal of $T$ is not generated by forms of degree $\leq \rho$.

Claim 1: We have $a_{1} \geq\lceil m / 6\rceil$.

Proof Claim 1. Since $d-1<\left(m^{2}+4 m\right) / 3$, it is sufficient to use that

$$
(5 m / 6+2)(5 m / 6+1) / 2=(5 m+12)(5 m+6) / 72 \geq\left(m^{2}+4 m\right) / 3 .
$$

Claim 2: For all integers $i \geq 2$ we have $a_{i} \geq\lceil m / 6\rceil+i$.

Proof Claim 2. Since $b_{1}+\cdots+b_{i-1} \leq m+2 i-1$ and $d-1<\left(m^{2}+4 m\right) / 3$, it is sufficient to use that

$$
(5 m / 6+i+2)(5 m / 6+i+1) / 2 \geq m+2 i+\left(m^{2}+4 m\right) / 3 .
$$

By Claims 1 and 2 and the assumption $m \geq 13$, we have $a_{i} \geq 3$ for all $i$. Hence $b_{i}=0$ for $i \gg 0$. Indeed we may take $b_{i}=0$ if $i>(\rho-m) / 2=c$

For each $i=1, \ldots, c$ take $o_{i} \in C \cap H_{i}$. Since $D \cap C=\emptyset$, we have $o_{i} \notin H_{j}$ if $i \neq j$. Fix a general line $L_{i 1}$ of $H_{i}$ containing $o_{i}$. If $b_{i} \leq 1$ set $L_{i j}=\emptyset$, $E_{i j}=\emptyset$ for all $j>1$ and $T_{i}:=L_{i 1}$. Now assume $b_{i} \geq 2$. Let $L_{i j}, 2 \leq j \leq b_{i}$, be general lines of $H_{i}$. Set $T_{i}:=L_{i 1} \cup \cdots \cup L_{i b_{i}}$. Let $E_{i} \subset H_{i}$ be the union of all points $L_{i h} \cap L_{i j}$ with $j \geq h+2$. Since $L_{i j}$ is a general line of $H_{i}$ if $j \geq 2$, we have $E_{i} \cap H_{t}=\emptyset$ if $t \neq i$ and $E_{i} \cap D=\emptyset$.

Since $h^{1}\left(N_{C}(-1)\right)=0$, as in [8, Théorème 1.5] (with $H$ instead of a quadric surface) we see that we may deform $C$ in such a way that all sets $C \cap H_{i}$ are general in $H_{i}$ and in particular they have the Hilbert function of a general subset of $H_{i}$ (if $c>1$ we do not (and if $c>2$ we cannot) assume that the set $C \cap\left(H_{1} \cup \cdots \cup H_{c}\right)$ is a general subset of $H_{1} \cup \cdots \cup H_{c}$ with the only restriction that its restriction to each $H_{i}$ has the same cardinality). We may also assume that $C \cap D=\emptyset$. By Claim 1 and we have $d \geq a_{i}$ for all $i$. Note that $E_{i} \cap E_{j}=\emptyset$ for all $i \neq j$. Set $\chi_{i}:=\cup_{o \in E_{i}} 2 o_{i}$ and $J_{i}:=T_{i} \cup \chi_{i}$, $Y_{i}:=C \cup J_{1} \cup \cdots \cup J_{i}, 1 \leq i \leq c$, and $Y:=Y_{c}$. Note that $T_{i}=J_{i}$ if $b_{i} \leq 1$, $\operatorname{Res}_{H_{1}}\left(Y \cup E_{1}\right)=Y, \operatorname{Res}_{H_{1}}\left(Y_{1}\right)=Y \cup E_{1}, \operatorname{Res}_{H_{i}}\left(Y_{i}\right)=Y_{i-1} \cup E_{i}$ for all $i=2, \ldots, c$, and $\operatorname{Res}_{H_{i}}\left(Y_{i-1} \cup E_{i}\right)=Y_{i-1}$. By the proof of [6, Example 2.1.1] each $J_{i}$ may be deformed to a chain of $\operatorname{deg}\left(T_{i}\right)$ lines in which the first line (in the ordering of the chain) is $L_{i 1}$ and all lines of the chain meets $D$. Hence 
we may find a deformed chain $I_{i}$ meeting quasi-transversally $C$ and at a unique point, $o_{i}$, and with $\operatorname{deg}\left(D \cap I_{i}\right)=\operatorname{deg}\left(T_{i}\right)$. Hence $Y$ is deformable to a smooth curve $X$ of degree $d$ and genus $g$ with $\operatorname{deg}(D \cap X)=d-\delta=\rho+1$. Using Remark 2.4 and the semicontinuity theorem for cohomology we get $h^{1}\left(\mathcal{O}_{X}(e)\right)=0$ and $h^{1}\left(N_{X}(-1)\right)=0$. Since $\operatorname{deg}(D \cap X)=\rho+1$, Remark 1.3 gives $h^{1}\left(\mathcal{I}_{X}(\rho-1)\right) \neq 0$. By Lemmas 2.1 and 2.3 we have $X \in \Gamma_{d-\delta}$. Thus to prove Theorem 1.1 for the case $\rho=\delta-d+1$, it is sufficient to prove that $h^{1}\left(\mathcal{I}_{X}(\rho)\right)=0$. By semicontinuity it is sufficient to prove that $h^{1}\left(\mathcal{I}_{Y}(\rho)\right)=0$.

Claim 3: We have $h^{1}\left(H_{c}, \mathcal{I}_{Y \cap H_{c}, H_{c}}(\rho)\right)=0$.

Proof Claim 3. Since $E_{i} \cap H_{c}=\emptyset$ for all $i<c$, we have $Y \cap H_{c}=T_{c} \cup\left(T_{1} \cup\right.$ $\left.\cdots \cup T_{c-1} \cup Y\right) \cap H_{c}$. Since $\left(T_{1} \cup \cdots \cup T_{c-1}\right) \cap H_{c} \subset D$ and $\operatorname{deg}\left(\left(T_{1} \cup \cdots \cup T_{c-1}\right) \cap\right.$ $\left.H_{c}\right) \leq \rho$, it is sufficient to prove that $h^{1}\left(H_{c}, \mathcal{I}_{C \cap\left(H_{c} \backslash T_{c}\right), H_{c}}\left(\rho-1-b_{c}\right)\right)=0$. This is true by the definition of $a_{c}$ and the assumption $b_{c} \leq a_{c}$.

Claim 4: We have $h^{1}\left(H_{c}, \mathcal{I}_{H_{c} \cap \operatorname{Res}_{H_{c}}(Y), H_{c}}(\rho-1)\right)=0$.

Proof Claim 4. We may apply Lemma 3.4, because $b_{c} \leq \rho-1$ and $H_{c} \cap C$ has the Hilbert function of a general subset of $H_{c}$ with cardinality $d$.

Claim 5: We have $h^{1}\left(\mathcal{I}_{Y}(\rho)\right)=0$.

Proof Claim 5. By Claims 3 and 4 it is sufficient to prove that $h^{1}\left(\mathcal{I}_{Y_{c-1}}(\rho-\right.$ $\left.b_{c}\right)=0$. We first check as in the proof of Claim 3 we proved first $h^{1}\left(H_{c-1}, \mathcal{I}_{Y_{c-1} \cap H_{c-1}, H_{c-1}}\left(\rho-b_{c}\right)\right)=0$. Then as in Claim 4 we proved that $h^{1}\left(H_{c-1}, \mathcal{I}_{\left(Y_{c-1} \cap H_{c-1}\right) \cup E_{c}, H_{c-1}}\left(\rho-b_{c}-1\right)\right)=0$ and then we continue using the planes $H_{c-2}, \ldots, H_{1}$.

(b) In this step we assume $\delta=\rho+1$ and $\rho-m$ odd, say $\rho=m+2 c+1$ for some integer $c$. Take $H_{1}, \ldots, H_{c}, b_{1}, \ldots, b_{c}$ as in step (a), but with respect to the integer $\rho-1$. We get a pair $(Y, D)$ with $Y$ a reducible curve $Y \in \Gamma_{\delta-1}$, $h^{1}\left(N_{Y}(-1)\right)=0, h^{1}\left(\mathcal{I}_{Y}(x)\right)=0$ for all $x \geq \rho-1$, and $D$ is a line with $\operatorname{deg}(D \cap Y)=\rho$. Take a general plane $H \supset D$ and let $D \subset H$ be a line containing exactly one point of $C \cap H$. Apply Lemma 3.7.

(c) Now we assume $\delta \geq \rho+2$. Set $\delta^{\prime}:=\delta-\rho-1$. Suppose we find an integer $\mu>m$ and $Y \in \Gamma\left(+\delta^{\prime}\right)$ with $h^{1}\left(\mathcal{I}_{Y}(x)\right)=0$ for every $x \geq m^{\prime}$ and 
$d+\delta^{\prime} \leq \frac{\mu^{2}+4 \mu+6}{3}$. We use $Y, d+\delta^{\prime}$ and $\mu$ instead of $C, d$ and $m$. We may use several times Lemma 3.8, if the integer $\mu-m$ is odd we also use once Lemma 3.7. Take for instance the first use of Lemma 3.8. from $m$ and $d$ to $m+2$ and $d+b$. Since $\frac{(m+2)^{2}+4(m+2)+6}{3}-\frac{m+2+4 m+6}{3}=8 m / 3+8 / 3>b$, the only restriction is the upper bound for $b$ assumed in Lemma 3.8. Since $d<\frac{m+2+4 m+6}{3}$ we may take any $b \leq\lceil m / 6\rceil$ and hence (taking $\mu=\rho-3$ ) we may take as $\delta^{\prime}$ any integer $\leq \sum_{i=0}^{\lfloor(\rho-3) / 2\rfloor-1}\lceil(m+2 i) / 6\rceil$ and in particular any integer $\leq\lfloor(\rho-3) / 2\rfloor\lceil m / 6\rceil$.

Remark 3.9. When $\delta \gg \rho+1$ the construction easily gives irreducible components $\Psi=\Gamma_{\delta}$ with smooth curves $A, B \in \Psi$ with $h^{1}\left(N_{A}(-1)\right)=$ $h^{1}\left(N_{B}(-1)\right)=0$ and with $r(A) \neq r(B)$, e.g. $r(A)=\rho$ and $r(B)=\rho-1$. In many cases we may find curves $A_{1}, \ldots, A_{k} \in \Psi$ with $r\left(A_{i}\right) \neq r\left(A_{j}\right)$ for all $i \neq j$, but we do not have a quantitative version of this observation.

\section{References}

1. E. Ballico, Ph. Ellia and C. Fontanari, Maximal rank of space curves in the Range A, arXiv: 1705.10113.

2. E. Carlini, M. V. Catalisano and A. V. Geramita, 3-dimensional sundials, Cent. Eur. J. Math., 9 (2011), no. 5, 949-971.

3. G. Ellingsrud and A. Hirschowitz, Sur le fibré normal des courbes gauches, C. R. Acad. Sci. Paris Sér. I Math., 299 (1984), no. 7, 245-248.

4. R. Hartshorne, On the classification of algebraic space curves, in: Vector bundles and differential equations (Nice 1979), 82-112, Progress in Math. 7 Birkhäuser, Boston 1980 .

5. R. Hartshorne, On the classification of algebraic space curves. II, in: Algebraic geometry, Bowdoin, 1985 (Brunswick, Maine, 1985), 145-164, Proc. Sympos. Pure Math., 46, Part 1, Amer. Math. Soc., Providence, RI, 1987.

6. R. Hartshorne and A. Hirschowitz, Droites en position générale dans $\mathbb{P}^{n}$, Algebraic Geometry, Proceedings, La Rábida 1981, 169-188, Lect. Notes in Math. 961, Springer, Berlin, 1982.

7. R. Hartshorne and A. Hirschowitz, Smoothing algebraic space curves, Algebraic Geometry, Sitges 1983, 98-131, Lecture Notes in Math. 1124, Springer, Berlin, 1985.

8. D. Perrin, Courbes passant par $m$ points généraux de $\mathbb{P}^{3}$, Bull. Soc. Math. France, Mémoire 28/29 (1987).

9. E. Sernesi, On the existence of certain families of curves, Invent. Math., 75 (1984), no. $1,25-57$. 